\title{
A serial mediation model of attentional engagement with thin bodies on body dissatisfaction: The role of appearance comparisons and rumination
}

Laura Dondzilo $^{\mathrm{a}, \mathrm{b}}$, Julian Basanovic ${ }^{\mathrm{a}, \mathrm{b}}$, Ben Grafton ${ }^{\mathrm{a}, \mathrm{b}}$, Jason Bell ${ }^{\mathrm{b}}$, Georgia Turnbull ${ }^{\mathrm{b}}$, and Colin

$$
\text { MacLeod }^{\mathrm{a}, \mathrm{b}}
$$

${ }^{a}$ Elizabeth Rutherford Memorial Centre for the Advancement of Research on Emotion, School of Psychological Science, University of Western Australia, Crawley, Western Australia, Australia.

${ }^{b}$ School of Psychological Science, University of Western Australia, Crawley, Western Australia, Australia.

Correspondence concerning this article should be addressed to Laura Dondzilo, School of Psychological Science, University of Western Australia (M304), 35 Stirling Highway, Crawley WA 6009, Australia. Phone: +61 408637 524. Email: laura.dondzilo@uwa.edu.au 


\begin{abstract}
The present study examined the associations among biased attentional responding to thinideal bodies, appearance comparisons, eating disorder-specific rumination, and body dissatisfaction. Sixty-seven females completed an attentional task capable of independently assessing biased attentional engagement with, and biased attentional disengagement from, images of thin-ideal bodies relative to images of non-thin bodies. Self-report measures of the other relevant constructs were also taken. Results revealed that a heightened tendency to engage in appearance comparisons was predicted by increased attentional engagement with thin-ideal bodies but not by impaired attentional disengagement from thin-ideal bodies. Moreover, a serial mediation analysis revealed that increased attentional engagement with thin-ideal bodies was associated with greater appearance comparison, which in turn was associated with greater eating disorder-specific rumination and consequently greater body dissatisfaction. The current findings suggest that increased attentional engagement with thin-ideal bodies might represent a pathway to body dissatisfaction, mediated by greater appearance comparison and eating-disorder specific rumination.
\end{abstract}


The majority of Australian women (86.9\%) report some level of dissatisfaction with their weight and shape (Mond et al., 2013). This is particularly concerning given that body dissatisfaction is a core contributor to the emergence of eating disorders (Stice, Gau, Rohde, \& Shaw, 2017; Stice, Marti, \& Durant, 2011; Stice \& Shaw, 2002). Internalisation of contemporary society's idealisation of thinness has been identified as a potent vulnerability factor for body dissatisfaction (Cafri, Yamamiya, Brannick, \& Thompson, 2006; Thompson \& Stice, 2001). As a consequence, researchers have been increasingly motivated to understand the cognitive factors that contribute to such dissatisfaction. One influential hypothesis is that cognitive biases, that operate to increase selectively processing of appearance-related stimuli representing the thin-ideal, may play a role in the onset and/or maintenance of body dissatisfaction (Jiang \& Vartanian, 2016; Rodgers \& DuBois, 2016).

Previous research has shown an association between an attentional bias to thin-ideal stimuli and body dissatisfaction in community samples of women (Cho \& Lee, 2013; Dondzilo, Rieger, Palermo, Byrne, \& Bell, 2017; Joseph et al., 2016; Moussally, Brosch, \& Van der Linden, 2016). Moreover, it has been proposed that eating disorder-specific rumination, which is the habitual tendency to engage in repetitive thinking about eating, shape, and weight concerns, may mediate the relationship between attentional bias to thin-ideal bodies and body dissatisfaction (Dondzilo et al., 2017). To test this hypothesis, Dondzilo et al. (2017) employed the conventional dot probe task to measure attentional selectivity (MacLeod, Mathews, \& Tata, 1986). In this study, undergraduate women were exposed to stimulus pairs, each comprising a body image (either thin-ideal or nonthin) and an abstract art image, for $500 \mathrm{~ms}$. A probe subsequently appeared in the location where either of the images were just displayed, and participants were required to make a discriminatory response to this probe. It was found that individuals who scored higher in eating disorder-specific rumination, as assessed by the Ruminative Response Scale for Eating Disorders (RRS-ED; Cowdrey \& Park, 2011), were disproportionately fast to discriminate probes replacing body images (relative to probes replacing abstract art images) when these were images of thin-ideal bodies. 
Furthermore, findings revealed that eating disorder-specific rumination served to mediate the relationship between attentional bias to thin-ideal bodies and body dissatisfaction. Nonetheless, two important questions were not addressed in this initial research, and resolving these issues will require methodological refinement.

First, this previous research did not take into consideration the potential role of appearance comparisons. The thin-ideal figure is extremely difficult, if not impossible, for most women to attain. As such, it is commonly theorised that an attentional bias to thin-ideal bodies triggers appearance comparisons that serve to highlight discrepancies between appearance ideals and one's own appearance, and in turn perpetuates dissatisfaction with one's own shape and weight (Rodgers \& DuBois, 2016). However, to date, no research has investigated whether attentional bias to thinideal bodies predicts the tendency to engage in appearance comparisons. The idea that certain types of appearance comparisons causally contribute to body image concerns is in line with sociocultural theory (Thompson, Heinberg, Altabe, \& Tantleff-Dunn, 1999) and is supported by empirical evidence (for meta-analyses see: Cafri et al., 2006; Myers \& Crowther, 2009). There is also evidence suggesting that social comparisons predict subsequent increases in rumination (Feinstein et al., 2013) and that induced eating disorder-specific rumination serves to exacerbate body image anxiety and dissatisfaction (Etu \& Gray, 2010; Rivière, Rousseau, \& Douilliez, 2018). This invites the possibility that attentional bias to thin-ideal bodies may increase the tendency to engage in appearance-based comparisons, which in turn inflates eating disorder-specific rumination, which increases body dissatisfaction.

The second crucial issue concerns whether elevated body dissatisfaction is characterised by increased attentional engagement with thin-ideal bodies, or reduced attentional disengagement from thin-ideal bodies. As has been pointed out elsewhere (Clarke, MacLeod, \& Guastella, 2013; Grafton, Watkins, \& MacLeod, 2012), the patterns of attentional bias revealed by the conventional dot probe assessment task cannot distinguish the tendency for a particular type of stimuli to selectively capture attention when presented distally to attentional focus (i.e. attentional 
engagement bias) from the tendency for this type of stimuli to selectively hold attention when presented proximal to attentional focus (i.e., attentional disengagement bias). Thus, because Dondzilo et al. (2017) employed the conventional dot probe assessment approach, it remains unclear which of these two types of attentional bias characterizes body dissatisfaction. Critically, distinguishing between biased attentional engagement and biased attentional disengagement has the potential both to advance theoretical understanding of body dissatisfaction and to inform intervention approaches. Theoretical conceptualisations of body dissatisfaction are likely to benefit from research that separately assesses these two aspects of attentional selectively to determine which is more involved in body dissatisfaction. The resulting new knowledge may, in turn, serve to identify the specific aspect of attentional selectivity that should be targeted in therapeutic interventions designed to attenuate the experience of body dissatisfaction by modifying the underlying patterns of biased information processing.

Theorists who have conceptually distinguished biased attentional engagement from biased attentional disengagement commonly suggest that these two facets of attentional selectivity might differentially contribute to various aspects of dysfunctional psychological experience (Grafton \& MacLeod, 2014; Rudaizky, Basanovic, \& MacLeod, 2014). Thus, it is possible that elevated body dissatisfaction may be a specific function of only one of these two facets of attentional bias. One such possibility is that elevated body dissatisfaction might be predicted by increased attentional engagement with thin-ideal bodies alone. An alternative possibility is that elevated body dissatisfaction may instead be predicted by reduced attentional disengagement from thin-ideal bodies alone. Of course, it is also possible that elevated body dissatisfaction may be equally well predicted by either form of attentional bias. In order to determine the validity of these three alternative possibilities, an assessment approach is required that permits the independent measurement of these two forms of attentional bias.

The Attentional Response to Distal vs. Proximal Emotional Information (ARDPEI; Grafton \& MacLeod, 2014) is an assessment task that satisfies the key methodological criteria considered 
necessary for the adequate differentiation of attentional engagement versus attentional disengagement (Clarke et al., 2013). Specifically, it introduces a means of securing initial attentional focus in a predetermined location, either distal from or proximal to the locus where the target stimulus images of interest are then presented. This permits separate assessment of the degree to which target stimuli presented distally from initial attentional focus selectively capture attention (thereby measuring bias in attentional engagement with such stimuli), and the degree to which target stimuli presented proximal to initial attentional focus selectively hold attention (thereby measuring bias in attentional disengagement from such stimuli). The capacity of this task to separately assess biased attentional engagement and biased attentional disengagement has been confirmed in prior studies investigating the contribution of each type of bias to variation in general ruminative disposition (Grafton, Southworth, Watkins, \& MacLeod, 2016; Southworth, Grafton, MacLeod, \& Watkins, 2016), anxiety vulnerability (Grafton \& MacLeod, 2014; Rudaizky et al., 2014), and Anorexia Nervosa (Jonker, Glashouwer, Hoekzema, Ostafin, \& de Jong, 2019).

The current study assessed attentional bias to thin-ideal bodies in undergraduate women by delivering the ARDPEI task, which yielded separate measures of biased attentional engagement with, and biased attentional disengagement from, thin-ideal vs non-thin bodies. Participants also completed self-report measures of appearance comparisons, eating disorder-specific rumination, and body dissatisfaction. Thus, the design of the present study made it possible to determine not only whether the tendency to exhibit appearance comparisons is predicted by an attentional bias to thin-ideal body images, but also to reveal whether it is increased attentional engagement with thinideal body images, reduced attentional disengagement from thin-ideal body images, or both, that predicts this tendency to engage in appearance comparison. Whichever is the case, the design also permits empirical evaluation of the serial mediation hypothesis, according to which such biased attention will indirectly predict body dissatisfaction through a pathway that involves two mediators. Specifically, the serial mediation hypothesis posits that biased attentional processing of thin-ideal bodies will directly predict appearance-based comparisons, appearance comparisons will directly 
predict eating disorder-specific rumination, and eating disorder-specific rumination will directly predict body dissatisfaction, giving rise to an indirect association between the biased attentional processing and body dissatisfaction.

\section{Method}

\section{Participants}

Sixty-seven undergraduate females from the University of Western Australia took part in the study in exchange for course credit. Given research showing differential patterns of attentional processing of body stimuli among varying weight categories (Lyu, Zheng, \& Wang, 2018; Warschburger, Calvano, Richter, \& Engbert, 2015), all participants were required to have a body mass index $\left(\mathrm{BMI}=\mathrm{kg} / \mathrm{m}^{2}\right)$ within the normal range (i.e., $\left.18.5-25 \mathrm{~kg} / \mathrm{m}^{2}\right)$. Ethical approval for this study was given by the University of Western Australia's Human Research Ethics Committee.

\section{Questionnaires}

Physical Appearance Comparison Scale-Revised (PACS-R). Tendency to engage in appearance comparisons was assessed using the 11-item PACS-R (Schaefer \& Thompson, 2014). Items are rated on a five-point Likert scale $(0=$ never, $4=$ always $)$ with higher scores indicating greater tendency to engage in appearance-based comparisons. The PACS-R has been previously validated within university student samples (Schaefer \& Thompson, 2014). Internal consistency in the current sample was high $(\alpha=.97)$.

Ruminative Response Scale for Eating Disorders (RRS-ED). Eating disorder specific rumination was measured using the nine-item RRS-ED (Cowdrey \& Park, 2011) Participants rate their tendency to experience rumination about eating, body shape, and/or weight concerns on a fourpoint scale $(1=$ almost never, $4=$ almost always $)$ with higher scores reflecting greater levels of eating disorder-specific rumination. The RRS-ED has demonstrated excellent psychometric properties within community samples of women (Cowdrey \& Park, 2011, 2012; Dondzilo et al., 2016). Internal consistency was high $(\alpha=.93)$ in the current sample. 
Body Shape Questionnaire (BSQ). Body dissatisfaction was measured using the 34-item BSQ (Cooper, Taylor, Cooper, \& Fairbum, 1987). Participants rate their tendency to experience concerns about body shape and feelings of fatness over the past four weeks on a six-point Likert scale $(1=$ never, 6 = always $)$ with higher scores reflecting higher levels of body dissatisfaction. The BSQ has shown excellent reliability and validity (Cooper et al., 1987; Rosen, Jones, Ramirez, \& Waxman, 1996). Internal consistency in the current sample was high $(\alpha=.98)$.

\section{Stimulus Images}

The attentional assessment task made use of 80 images, which were of two different types. Half of the images (40) were "representational", in that they depicted female bodies, while the other half (40) were "non-representational" images, in that they depicted abstract art. The representational images were the same stimuli employed by Dondzilo et al. (2017). Half of the representational images (20) were thin bodies, estimated to be bordering on underweight, which is consistent with the thin-ideal. The remaining 20 were non-thin bodies, estimated to be in the overweight/mildly obese weight range. Each image focused on a weight and/or shape relevant area, such as the abdomen and thighs.

\section{Attentional Response to Distal vs. Proximal Emotional Information (ARDPEI) Task}

The ARDPEI task (Grafton \& MacLeod, 2014) was used to assess biased attentional engagement with, and biased attentional disengagement from, thin-ideal body images relative to the non-thin body images. Task specifications (i.e., number of trials, counterbalancing, and stimulus exposure duration) followed Dondzilo et al. (2017) given that the use of these task parameters in this prior work served to reveal the associations between attentional bias towards thin-ideal bodies, eating disorder-specific rumination, and body dissatisfaction, that the present study was intended to further illuminate.

Each trial commenced with the $1000 \mathrm{~ms}$ presentation of two white rectangle outlines, each measuring $175 \mathrm{~mm}$ x $115 \mathrm{~mm}$, one on each side of the screen. The centres of the left and right rectangles were positioned approximately $125 \mathrm{~mm}$ to the left and right of the screen centre, 
respectively. A smaller red rectangular outline, measuring $25 \mathrm{~mm}$ x $25 \mathrm{~mm}$, appeared in either of the bigger white rectangles with equal frequency. Participants were required to initially focus their attention within this red rectangle. $1000 \mathrm{~ms}$ later, an anchor cue briefly appeared (200 ms) within this attended region. This was a small $(5 \mathrm{~mm})$ red line, which with equal frequency was either horizontal or vertical in orientation, and participants were required to note its orientation. Immediately thereafter, an image pair consisting of a representational image (i.e., thin or non-thin body) and non-representational image (i.e., abstract art) was presented for $500 \mathrm{~ms}$, one image appearing in each of the two screen regions denoted by the white rectangles. On half the trials, the representational image appeared in the opposite location to that where participant's initial attention had been anchored at the beginning of the trial, and these trials served to assess selective attentional engagement with distal images of thin compared to non-thin bodies. On the other half of the trials, the representational image appeared in the same location where participant's initial attention had been anchored at the beginning of the trial, and these trials served to assess selective attentional disengagement from proximal images of thin compared to non-thin bodies.

Attentional distribution between the two loci at the end of the $500 \mathrm{~ms}$ image display was assessed by then presenting a target probe in either of the two screen regions with equal probability. The target probe was also a small $(5 \mathrm{~mm})$ red line, either horizontal or vertical in orientation. Participants were required to indicate, as quickly as possible, whether the orientation of the target probe matched that of the previously exposed anchor cue, which was the case on $50 \%$ of the trials. Responses were registered by pressing either the left or right mouse button to respectively indicate that the target probe orientation did or did not match anchor probe orientation. The latency to make this probe discrimination response was recorded, as was its accuracy, and it was assumed that participants would be faster to accurately respond to probes that appeared in the attended screen location that in the unattended screen location. Following detection of this response, the screen was cleared for $1000 \mathrm{~ms}$, and then the next trial commenced. 
The task delivered two blocks of 160 trials. In one block the representational images were thin bodies, and in the other block they were non-thin bodies. The order of these blocks was counterbalanced across participants. Within each block, order of trial condition was randomised for each participant.

\section{Calculation of Attentional Engagement and Disengagement Bias Index Scores.}

Latencies to make correct responses to the target probe under the differing conditions of the ARDPEI task were used to compute two indices of attentional bias, each reflecting increased attention to thin-ideal bodies relative to non-thin bodies, but one representing increased attentional engagement with thin-ideal bodies relative to non-thin bodies presented distally from initial attentional focus, and the other representing reduced attentional disengagement from thin-ideal bodies relative to non-thin bodies presented proximally to initial attentional focus. Before computing the two attentional bias scores, probe discrimination latencies that fell further than 2.58 standard deviations from the participant's mean probe discrimination latency for each experimental condition were eliminated, in line with previous practice (Grafton \& MacLeod, 2014; Jonker et al., 2019; Rudaizky et al., 2014). Finally, probe discrimination latencies that were < $200 \mathrm{~ms}$ (i.e., anticipation errors) were also excluded.

The engagement bias index score was then computed from those trials on which representational image appeared in the opposite locus from that in which the anchor cue had secured the participants' initial attentional focus. Specifically, this index reflected the degree to which the latency to discriminate target probes in the location of the body image (presented distally from initial attentional focus), compared to target probes in the location of the abstract image presented proximally to initial attentional focus, was disproportionately speeded when this representational image was a thin body rather than a non-thin body. This required first computing, for trials on which representational images were presented distally from initial attentional focus, the magnitude of relative speeding to probes in the locus of the representational image relative to those in the locus the accompanying abstract image, separately for trials on which the representational 
image was a thin body and for trials on which it was a non-thin body; then subtracting the latter value from the former value. A larger score represents increased attentional engagement with distal images of thin bodies relative to non-thin bodies. Mathematically, this can be expressed as:

Engagement bias index $=$ (anchor cue distal from thin body image: response latency for target probe in locus of abstract image minus response latency for target probe in locus of thin body image) minus (anchor cue distal from non-thin body image: response latency for target probe in locus of abstract image minus response latency for target probe in locus of non-thin body image)

In contrast, the disengagement bias index score was computed from those trials on which the representational image appeared in the same locus as that in which the anchor cue had secured the participants' initial attentional focus. This index reflected the degree to which the latency to discriminate target probes in the location of the body image (presented proximally to initial attentional focus), compared to target probes in the location of the abstract image presented distally to initial attentional focus, was disproportionately speeded when this representational image was a thin body rather than non-thin body. This required first computing, now for trials on which representational images were presented proximally to initial attentional focus, the magnitude of relative speeding to probes in the locus of the body relative to those in the locus the accompanying abstract image, separately for trials on which the body image was thin and for trials on which the body image was non-thin; then subtracting the latter value from the former value. A larger score represents reduced attentional disengagement from the proximal images of thin bodies relative to non-thin bodies. Mathematically, this can be expressed as:

Disengagement bias index $=($ anchor cue proximal to thin body image: response latency for target probe in locus of abstract image minus response latency for target probe in locus of thin body image) minus (anchor cue proximal to non-thin body image: response latency for target probe in locus of abstract image minus response latency for target probe in locus of non-thin body image). 


\section{Procedure}

Participants were tested individually in a quiet room. After providing informed consent, each participant was given instructions for the ARDPEI task, then completed by a short practice comprising 32 trials that employed only abstract art stimuli, not used within the assessment version of the task. Next, participants completed the assessment version of the ARDPEI task, followed by the PACS-R, the RRS-ED and the BSQ. Finally, participants' height and weight were measured to enable calculation of BMI. At the end of the session, the participant was fully debriefed.

\section{Results}

The data were first screened for missing values and outliers. One participant had a corrupted data file and a further three participants were identified as outliers in terms of their probe discrimination accuracy on the ARDPEI task (i.e., accuracy score fell more than 2.58 standard deviations below the mean), which resulted in the elimination of these four participants. Accuracy was very high across the remaining 63 participants, averaging $95.46 \%$ accuracy. Descriptive statistics for age, BMI, engagement bias index scores, disengagement bias index scores, PACS-R scores, RRS-ED scores, and BSQ scores are reported in Table 1.

\section{Was the proposed serial mediation hypothesis with engagement bias as the antecedent supported?}

The serial mediation hypothesis predicts that increased attentional engagement with thinideal body images will be associated with a greater tendency to engage in appearance comparisons, which in turn will be associated with greater eating disorder-specific rumination, and consequently greater body dissatisfaction. Results of the first order correlations revealed that engagement bias index scores, reflecting increased attentional engagement with thin-ideal body images relative to non-thin body images, were positively correlated with appearance comparisons scores $(r=.34, p=$ .006), appearance comparisons scores were positively correlated with eating disorder-specific rumination scores $(r=.78, p<.001)$, and eating disorder-specific rumination scores were positively correlated with body dissatisfaction scores $(r=.90, p<.001)$. 
To determine whether increased attentional engagement with thin-ideal bodies was indirectly associated with greater body dissatisfaction, through appearance comparisons and eating disorder-specific rumination, a serial mediation analysis was conducted (see Figure 1). A percentile bootstrap confidence interval (CI) for this hypothesised indirect effect based on 5,000 bootstrap samples was entirely above zero $(\beta=.16, S E=.07,95 \% \mathrm{CI}=.03, .31)$, indicating a statistically significant indirect effect. Neither the direct effect $(\beta=-.06, S E=.05,95 \% \mathrm{CI}=-.17, .04)$ nor the total effect $(\beta=.18, S E=.13,95 \% \mathrm{CI}=-.08, .43)$ of engagement bias on body dissatisfaction was statistically significant. Controlling for BMI did not modify this pattern of results. The model accounted for $86 \%$ of the variance in body dissatisfaction, $F(3,59)=121.97, p<.001$.

\section{Was the proposed serial mediation hypothesis with disengagement bias as the antecedent supported?}

Although disengagement bias index scores did not correlate with any of the key variables included in the serial meditation model (all $p$ 's $>.12$ ), this does not preclude the possibility that biased attentional disengagement might indirectly associate with body dissatisfaction. Thus, we additionally tested the proposed serial mediation model with disengagement bias as the antecedent (see Figure 2). A percentile bootstrap CI for this hypothesised indirect effect based on 5,000 bootstrap samples was not above zero $(\beta=-.01, S E=.06,95 \% \mathrm{CI}=-.11, .13)$, indicating the absence of a statistically significant indirect effect. Additionally, there was no statistically significant direct effect of disengagement bias on body dissatisfaction $(\beta=.05, S E=.05,95 \% \mathrm{CI}=$ $-.05, .15)$. Controlling for BMI did not modify this pattern of results. The model accounted for $86 \%$ of the variance in body dissatisfaction, $F(3,59)=120.53, p<.001$. 
Table 1

Descriptive statistics for age, BMI, Engagement Bias Index scores, Disengagement Bias Index scores, PACS-R scores, RRS-ED scores, and BSQ scores.

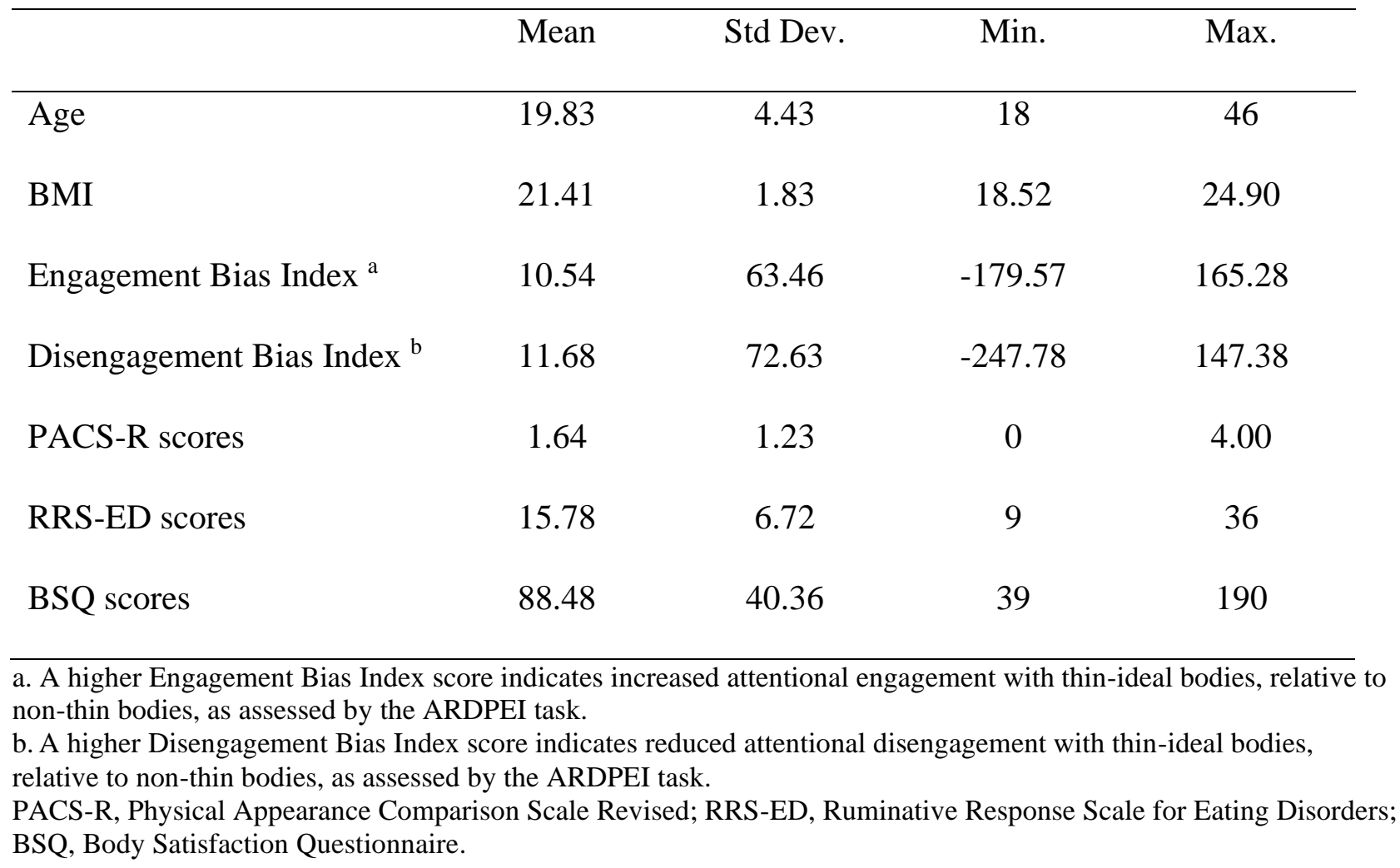




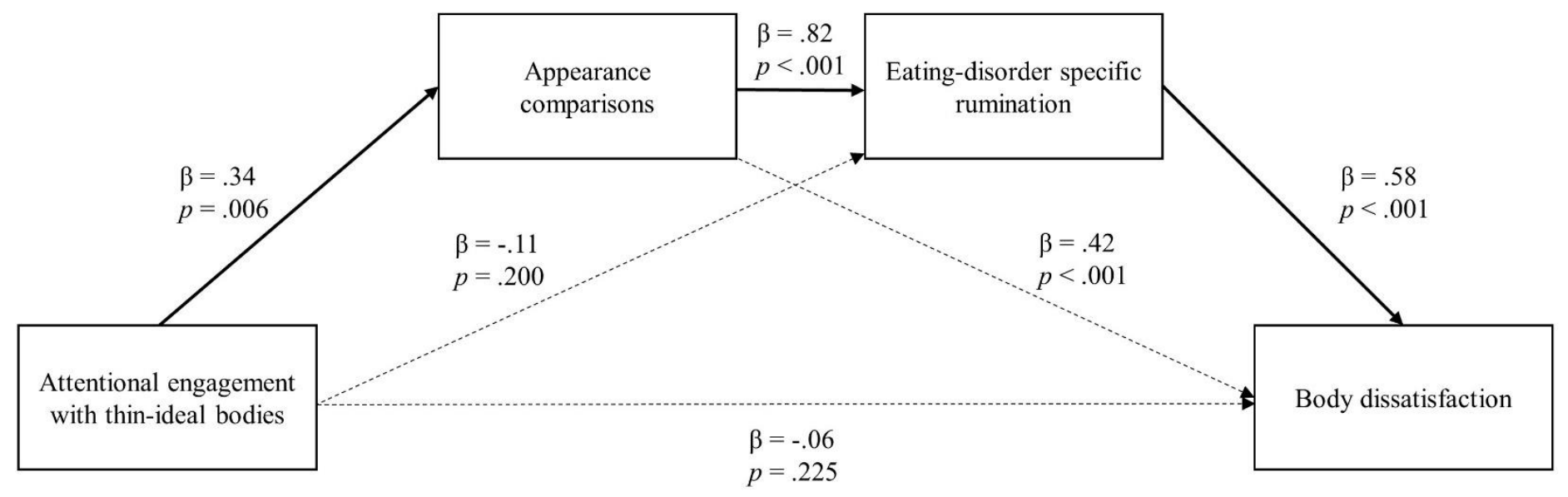

Figure 1. Serial mediation model representing the mediating effects of appearance comparisons and eating disorder-specific rumination on attentional engagement bias with thin-ideal bodies and body dissatisfaction in healthy-weight females. All regression coefficients are standardised. The bolded lines represent the hypothesised serial mediation effect. The dotted lines represent alternative pathways. 


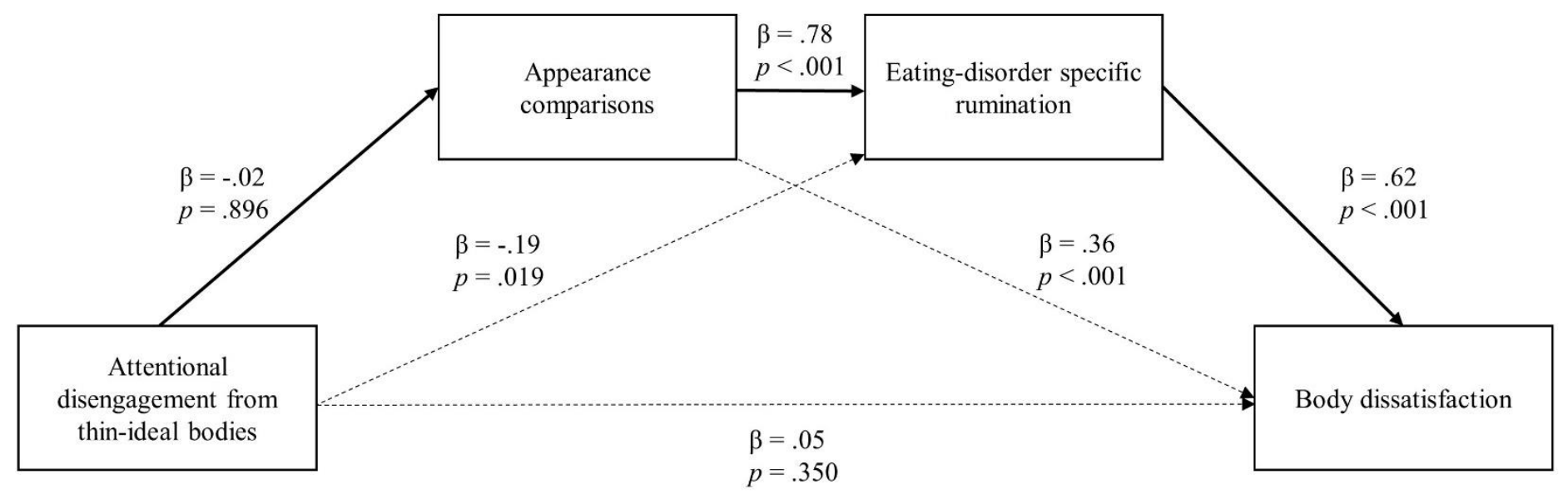

Figure 2. Serial mediation model representing the mediating effects of appearance comparisons and eating disorder-specific rumination on attentional disengagement bias from thin-ideal bodies and body dissatisfaction in healthy-weight females. All regression coefficients are standardised. The bolded lines represent the hypothesised serial mediation effect. The dotted lines represent alternative pathways. 


\section{Discussion}

The current study sought to investigate the serial mediation hypothesis, according to which attentional bias towards thin-ideal bodies drives more frequent appearance comparisons, which in turn drives greater eating disorder-specific rumination, which in turn drives greater body dissatisfaction, resulting in an indirect association between this attentional bias and body dissatisfaction. Our results provide support for this hypothesised indirect effect of attentional bias on body dissatisfaction, for one of the two indices of attentional bias assessed. Specifically, this serial mediation effect was confirmed when examining the relationship between biased attentional engagement with thin-ideal body images and body dissatisfaction. The serial mediation effect was not observed with biased attentional disengagement as the antecedent. Thus, the obtained pattern of results suggests that increased attentional engagement with thin-ideal bodies, but not reduced attentional disengagement from thin-ideal bodies, predicts engagement in appearance comparison, which in turn predicts eating disorder-specific rumination, which predicts body dissatisfaction, with this serial mediation resulting in a significant indirect association between increased attentional engagement with thin-ideal bodies and body dissatisfaction.

This is the first time, to our knowledge, that the possible contribution to appearance comparisons made by biased attention for appearance-related information has differentiated the variance explained by biased patterns of attentional engagement and disengagement. The current paradigm permitted the dissociation between these two types of attentional bias by anchoring initial attentional focus in a predetermined location, before the presentation of differentially valenced information either distal or proximal to this initial attentional focus. Findings obtained in the current study were novel in showing that the tendency to engage in appearance comparisons was predicted by increased attentional engagement with thin-ideal bodies and not by impaired attentional disengagement from thin-ideal bodies.

The current study is also the first to examine the mediating role of appearance comparisons, in addition to eating disorder-specific rumination, in the relationship between biased attentional 
responding to body images and body dissatisfaction. The current study revealed no evidence of a first-order association between either attentional engagement with thin-ideal bodies or attentional disengagement from thin-ideal bodies and body dissatisfaction. Instead, we found only an indirect association, whereby heightened attentional engagement with thin-ideal bodies alone predicted body dissatisfaction through the sequential mediators of appearance comparisons and eating disorder-specific rumination. This highlights the importance of considering these mediating factors, implicated in the proposed model under test. Due to the inclusion of these proposed mediators in our study design, and the methodological refinement that enabled us to discretely assess bias attentional engagement with, and disengagement from, thin-ideal bodies, the present study has moved beyond the limitations of previous research (Dondzilo et al., 2017) to enable a more complete understanding of the specific attentional mechanisms and mediators underpinning the relationship between biased attentional processing of thin-ideal bodies and body dissatisfaction.

Of course, it is important to acknowledge that the presently observed associations do not permit conclusions concerning causality. Specifically, the observed serial mediation effect does not dictate that each variable in the mediational pathway necessarily causally impacts on the next variable in this pathway. There would be value in empirically determining whether biased attentional engagement with thin-ideal bodies plays a causal role in indirectly driving body dissatisfaction through the presently observed mediational pathway. This will require developing training variants of ARDPEI task, specifically configured to induce biased attentional engagement with thin-ideal bodies, to determine whether the direct modification of this attentional engagement bias serves to alter appearance comparisons. The outcome of such attentional bias modification studies will serve to determine whether biased attentional engagement with thin-ideal bodies causally contributes to the tendency to engage in appearance comparison. If such a causal relationship is established, then this will provide a firm justification for developing and evaluating therapeutic interventions designed to target this engagement bias, with the aim of directly reducing 
engagement in appearance comparison, to determine whether this serves to indirectly attenuate body dissatisfaction.

Moreover, it remains unknown whether the pattern of biased attentional engagement implicated in elevated body dissatisfaction resulted from automatic or controlled attentional selectivity. More specifically, the current study only examined attentional engagement and disengagement using a stimuli exposure duration of $500 \mathrm{~ms}$. While $500 \mathrm{~ms}$ is the most widely used stimulus exposure duration, it permits the operation of both automatic and controlled patterns of attentional selectivity (Holender, 1986; Mogg, Bradley, \& Williams, 1995). In order to distinguish automatic from controlled processing, some previous implementations of the ARDPEI task have employed exposure durations as short as $100 \mathrm{~ms}$ (Jonker et al., 2019), and as long as $1000 \mathrm{~ms}$ (Grafton et al., 2016). By comparing the outcomes obtained when future variants of the present study employ stimuli exposure durations of 100,500 , and $1000 \mathrm{~ms}$, it will become possible to determine whether the increased attentional engagement with thin-ideal bodies presently found to characterise heightened body dissatisfaction is driven by automatic or controlled attentional processing.

Several other issues could also be usefully addressed by future research. Namely, it has been proposed that appearance comparisons which involve subjectively comparing one's own body to those of women whose bodies are considered thinner than one's own, referred to as upwards comparisons, might be especially problematic with respect to involvement in body dissatisfaction (Myers \& Crowther, 2009; Thompson et al., 1999). The currently employed measure of appearance comparison, however, did not differentiate general vs. upwards appearance comparisons. We recommend that future extensions of this work include a measure of appearance comparison which differentiates between these two different types of comparisons in order to determine whether it is appearance comparisons in general, or only upwards comparisons, that is implicated in the mediational pathway established by the present study. Furthermore, it remains uncertain whether the mediational model supported by the present findings, concerning the factors that contribute to 
variation in body dissatisfaction across an unselected sample of healthy-weight females, can also account for the elevated levels of body dissatisfaction experienced by individuals with eating disorders, compared to non-clinical controls.

\section{Conclusion}

We encourage fellow investigators to address these issues in future extensions of the presently reported work. For the moment, the findings obtained in the current study serve to advance understanding of the cognitive underpinnings of body dissatisfaction in healthy-weight women. Specifically, the findings demonstrate that, heightened tendency to engage in appearance comparisons is characterised by increased attentional engagement with thin-ideal bodies, relative to non-thin bodies. Moreover, increased attentional engagement with thin-ideal bodies was found to be indirectly associated with increased body dissatisfaction through appearance comparisons and eating disorder-specific rumination. These findings suggest that increased attentional engagement with thin-ideal bodies may represent a potential pathway to body dissatisfaction. 


\section{References}

Bocage-Barthélémy, Y., Chatard, A., Jaafari, N., Tello, N., Billieux, J., Daveau, E., \& Selimbegović, L. (2018). Automatic social comparison: Cognitive load facilitates an increase in negative thought accessibility after thin ideal exposure among women. PLOS ONE, 13(3), e0193200. https://doi.org/10.1371/journal.pone.0193200

Brown, A., \& Dittmar, H. (2005). Think “Thin” and Feel Bad: The Role of Appearance Schema Activation, Attention Level, and Thin-Ideal Internalization for Young Women's Responses to Ultra-Thin Media Ideals. Journal of Social and Clinical Psychology, 24(8), 1088-1113. https://doi.org/10.1521/jscp.2005.24.8.1088

Cafri, G., Yamamiya, Y., Brannick, M., \& Thompson, J. K. (2006). The Influence of Sociocultural Factors on Body Image: A Meta-Analysis. Clinical Psychology: Science and Practice, 12(4), 421-433. https://doi.org/10.1093/clipsy.bpi053

Chatard, A., Bocage-Barthélémy, Y., Selimbegović, L., \& Guimond, S. (2017). The woman who wasn't there: Converging evidence that subliminal social comparison affects self-evaluation. Journal of Experimental Social Psychology, 73, 1-13. https://doi.org/10.1016/j.jesp.2017.05.005

Cho, A., \& Lee, J. H. (2013). Body dissatisfaction levels and gender differences in attentional biases toward idealized bodies. Body Image, 10(1), 95-102. https://doi.org/10.1016/j.bodyim.2012.09.005

Clarke, P. J. F., MacLeod, C., \& Guastella, A. J. (2013). Assessing the role of spatial engagement and disengagement of attention in anxiety-linked attentional bias: a critique of current paradigms and suggestions for future research directions. Anxiety, Stress and Coping, 26(1), 119. https://doi.org/10.1080/10615806.2011.638054

Cooper, P. J., Taylor, M. J., Cooper, Z., \& Fairbum, C. G. (1987). The development and validation of the body shape questionnaire. International Journal of Eating Disorders, 6(4), 485-494. https://doi.org/10.1002/1098-108X(198707)6:4<485::AID-EAT2260060405>3.0.CO;2-O 
Cowdrey, F. A., \& Park, R. J. (2011). Assessing rumination in eating disorders: Principal component analysis of a minimally modified ruminative response scale. Eating Behaviors, 12(4), 321-324. https://doi.org/10.1016/j.eatbeh.2011.08.001

Cowdrey, F. A., \& Park, R. J. (2012). The role of experiential avoidance, rumination and mindfulness in eating disorders. Eating Behaviors, 13(2), 100-105. https://doi.org/10.1016/j.eatbeh.2012.01.001

Dondzilo, L., Rieger, E., Palermo, R., Byrne, S., \& Bell, J. (2016). Association between rumination factors and eating disorder behaviours in young women. Advances in Eating Disorders, 4(1), 84-98. https://doi.org/10.1080/21662630.2015.1118642

Dondzilo, L., Rieger, E., Palermo, R., Byrne, S., \& Bell, J. (2017). The mediating role of rumination in the relation between attentional bias towards thin female bodies and eating disorder symptomatology. PLOS ONE, 12(5), e0177870. https://doi.org/10.1371/journal.pone.0177870

Etu, S. F., \& Gray, J. J. (2010). A preliminary investigation of the relationship between induced rumination and state body image dissatisfaction and anxiety. Body Image, 7(1), 82-85. https://doi.org/10.1016/j.bodyim.2009.09.004

Feinstein, B. A., Hershenberg, R., Bhatia, V., Latack, J. A., Meuwly, N., \& Davila, J. (2013). Negative social comparison on Facebook and depressive symptoms: Rumination as a mechanism. Psychology of Popular Media Culture, 2(3), 161-170. https://doi.org/10.1037/a0033111

Grafton, B., \& MacLeod, C. (2014). Enhanced probing of attentional bias: The independence of anxiety-linked selectivity in attentional engagement with and disengagement from negative information. Cognition and Emotion, 28(7), 1287-1302. https://doi.org/10.1080/02699931.2014.881326

Grafton, B., Southworth, F., Watkins, E., \& MacLeod, C. (2016). Stuck in a sad place: Biased attentional disengagement in rumination. Emotion, 16(1), 63-72. 
https://doi.org/10.1037/emo0000103

Grafton, B., Watkins, E., \& MacLeod, C. (2012). The ups and downs of cognitive bias: Dissociating the attentional characteristics of positive and negative affectivity. Journal of Cognitive Psychology, 24(1), 33-53. https://doi.org/10.1080/20445911.2011.578066

Holender, D. (1986). Semantic activation without conscious identification in dichotic listening, parafoveal vision, and visual masking: A survey and appraisal. Behavioral and Brain Sciences, 9(1), 1-23. https://doi.org/10.1017/S0140525X00021269

Jiang, M. Y. W., \& Vartanian, L. R. (2016). The role of memory in the relationship between attention toward thin-ideal media and body dissatisfaction. Eating and Weight Disorders Studies on Anorexia, Bulimia and Obesity, 21(1), 57-64. https://doi.org/10.1007/s40519-0150196-x

Jonker, N. C., Glashouwer, K. A., Hoekzema, A., Ostafin, B. D., \& de Jong, P. J. (2019). Attentional engagement with and disengagement from food cues in Anorexia Nervosa. Behaviour Research and Therapy, 114(December 2018), 15-24. https://doi.org/10.1016/j.brat.2019.01.003

Joseph, C., LoBue, V., Rivera, L. M., Irving, J., Savoy, S., \& Shiffrar, M. (2016). An attentional bias for thin bodies and its relation to body dissatisfaction. Body Image, 19, 216-223. https://doi.org/10.1016/j.bodyim.2016.10.006

Lyu, Z., Zheng, P., \& Wang, Z. (2018). Time Course of Attentional Biases Toward Body Shapes in Women Who Are Overweight or Obese. Cognitive Therapy and Research. https://doi.org/10.1007/s10608-018-9978-6

MacLeod, C., Mathews, A., \& Tata, P. (1986). Attentional bias in emotional disorders. Journal of Abnormal Psychology, 95(1), 15-20. https://doi.org/10.1037/0021-843X.95.1.15

Mogg, K., Bradley, B. P., \& Williams, R. (1995). Attentional bias in anxiety and depression: The role of awareness. British Journal of Clinical Psychology, 34(1), 17-36. https://doi.org/10.1111/j.2044-8260.1995.tb01434.x 
Moussally, J. M., Brosch, T., \& Van der Linden, M. (2016). Time course of attentional biases toward body shapes: The impact of body dissatisfaction. Body Image, 19, 159-168. https://doi.org/10.1016/j.bodyim.2016.09.006

Myers, T. A., \& Crowther, J. H. (2009). Social comparison as a predictor of body dissatisfaction: A meta-analytic review. Journal of Abnormal Psychology, 118(4), 683-698. https://doi.org/10.1037/a0016763

Rivière, J., Rousseau, A., \& Douilliez, C. (2018). Effects of induced rumination on body dissatisfaction: Is there any difference between men and women? Journal of Behavior Therapy and Experimental Psychiatry, 61(September 2017), 1-6.

https://doi.org/10.1016/j.jbtep.2018.05.005

Rodgers, R. F., \& DuBois, R. H. (2016). Cognitive biases to appearance-related stimuli in body dissatisfaction: A systematic review. Clinical Psychology Review, 46, 1-11. https://doi.org/10.1016/j.cpr.2016.04.006

Rosen, J. C., Jones, A., Ramirez, E., \& Waxman, S. (1996). Body shape questionnaire: Studies of validity and reliability. International Journal of Eating Disorders, 20(3), 315-319. https://doi.org/10.1002/(SICI)1098-108X(199611)20:3<315::AID-EAT11>3.0.CO;2-Z

Rudaizky, D., Basanovic, J., \& MacLeod, C. (2014). Biased attentional engagement with, and disengagement from, negative information: Independent cognitive pathways to anxiety vulnerability? Cognition and Emotion, 28(2), 245-259. https://doi.org/10.1080/02699931.2013.815154

Schaefer, L. M., \& Thompson, J. K. (2014). The development and validation of the Physical Appearance Comparison Scale-Revised (PACS-R). Eating Behaviors, 15(2), 209-217. https://doi.org/10.1016/j.eatbeh.2014.01.001

Southworth, F., Grafton, B., MacLeod, C., \& Watkins, E. (2016). Heightened ruminative disposition is associated with impaired attentional disengagement from negative relative to positive information: support for the "impaired disengagement" hypothesis. Cognition and 
Emotion, 9931(February), 1-13. https://doi.org/10.1080/02699931.2015.1124843

Stice, E., Gau, J. M., Rohde, P., \& Shaw, H. (2017). Risk factors that predict future onset of each DSM-5 eating disorder: Predictive specificity in high-risk adolescent females. Journal of Abnormal Psychology, 126(1), 38-51. https://doi.org/10.1037/abn0000219

Stice, E., Marti, C. N., \& Durant, S. (2011). Risk factors for onset of eating disorders: Evidence of multiple risk pathways from an 8-year prospective study. Behaviour Research and Therapy, 49(10), 622-627. https://doi.org/10.1016/j.brat.2011.06.009

Stice, E., \& Shaw, H. E. (2002). Role of body dissatisfaction in the onset and maintenance of eating pathology. Journal of Psychosomatic Research, 53(5), 985-993. https://doi.org/10.1016/S0022-3999(02)00488-9

Thompson, J. K., Heinberg, L. J., Altabe, M., \& Tantleff-Dunn, S. (1999). Exacting beauty: Theory, assessment, and treatment of body image disturbance. American Psychological Association. Washington: American Psychological Association. https://doi.org/10.1037/10312-000

Thompson, J. K., \& Stice, E. (2001). Thin-Ideal Internalization: Mounting Evidence for a New Risk Factor for Body-Image Disturbance and Eating Pathology. Current Directions in Psychological Science, 10(5), 181-183. https://doi.org/10.1111/1467-8721.00144

Want, S. C. (2009). Meta-analytic moderators of experimental exposure to media portrayals of women on female appearance satisfaction: Social comparisons as automatic processes. Body Image, 6(4), 257-269. https://doi.org/10.1016/j.bodyim.2009.07.008

Warschburger, P., Calvano, C., Richter, E. M., \& Engbert, R. (2015). Analysis of Attentional Bias towards Attractive and Unattractive Body Regions among Overweight Males and Females: An Eye-Movement Study. PLOS ONE, 1O(10), e0140813. https://doi.org/10.1371/journal.pone.0140813 
